\section{Diplopia following ID block}

Sir, Scott et al. (BDJ 2007; 202: 32-33) describe an interesting case of double vision and local cutaneous vasoconstriction in the upper lip, just lateral to the philtrum, following inferior dental and long buccal nerve blocks. As the authors suggest, some of the more obvious anatomical explanations seem unlikely. They report deep anaesthesia with the patient feeling 'odd' after administration of local anaesthetic, which suggests there was direct leakage of anaesthetic into the vascular system, despite aspiration to try and avoid this. A more likely explanation for the diplopia is that anaesthetic entered veins communicating with the pterygoid venous plexus surrounding the pterygoid muscles. This plexus anastomoses freely both with emissary veins that pass through the foramina spinosum and ovale (occasionally also through a foramen of their own, the foramen of Vesalius). All of this valveless system of veins communicates with the cavernous venous sinus intracranially, and through this, with veins of the orbital cavities, forehead and face.

The tough lateral wall of the cavernous sinus, to which the dura mater of the middle cranial fossa is tightly bound, contains within it cranial nerves III, IV, Vi and Vii. However, the VIth nerve runs free within the venous blood of the cavernous sinus on the lateral aspect of the internal carotid artery, which itself also courses through the venous blood accompanied by a plexus of sympathetic nerves destined for the face and forehead - and which are vasomotor and secretomotor to sweat glands in the skin. Local anaesthetic reaching the cavernous sinus is, therefore, immediately in contact with the VIth nerve, which is likely to be affected by it, resulting in either partial or sometimes complete inability to abduct the eye until it clears.

The blanching of the upper lip is harder to explain but the deep facial vein runs in the cheek with the long buccal nerve and artery. The rich anastomosis of arteries around the upper lip would suggest it is unlikely that any one of them would have a discrete cutaneous distribution. Nonetheless, in this patient it remains a possibility that the buccal artery may indeed extend anteriorly through the cheek and into the skin of the upper lip. Anaesthetic and vasoconstrictor in the buccal artery would lead to immediate vasoconstriction. Anaesthetic in the deep facial vein here could again also easily pass back to reach the cavernous sinus. Whether this poor patient experienced two rare bouts of bad luck, that each resulted in a separate sign, or just one associated with the long buccal nerve block, is debatable, but these rare and alarming occurrences remind us of why we all spent so long learning head and neck anatomy. (The best account of these kind of anatomical details is undoubtedly Hollinshead W H. Anatomy for surgeons, volume 1, head and neck. Philadelphia: Harper Row, 1982.)

C. Dean

London

Editor's note: Regrettably, this letter was printed with a number of inadvertent errors in BDJ 2007; 202: 237, due to typographical misunderstandings. ' $\mathrm{V} i$ ' and 'Vii' were given as 'VI' and 'VII'. The version printed here is the correct version. We would like to apologise to Professor Dean, and would also like to thank Miss Sheila Lumley for bringing it to our attention. Notwithstanding the correction of these errors, there are other elements of Professor Dean's letter and the subject in general that Miss Lumley would like to raise (see below).

Sir, in respect of the article by Scott et al. (BDJ 2007; 202: 32-33) and the subsequent response from Professor Dean (BDJ 2007; 202: 237) I believe there are still factual errors.

Firstly, cranial nerve VII (facial) does not enter the cavernous sinus at all and secondly, only nerves III (oculomotor), IV (trochlear), and Va (ophthalmic division of trigeminal) lie in the lateral wall. In addition $\mathrm{Vb}$ (maxillary division of trigeminal) enters the cavernous sinus for a short distance before leaving

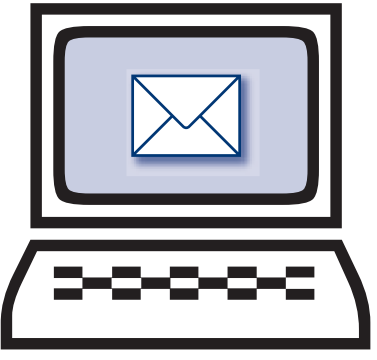

it to pass thorough foramen rotundum. VI (abducent) does not lie in the lateral wall but passes through the centre of the sinus with the internal carotid artery. I understand that these errors arose from an editorial misinterpretation (see above) in which Vi and Vii were confused for VI and VII respectively. In response to this, I would suggest that the designations Va, $\mathrm{Vb}$ and $\mathrm{Vc}$ are more appropriate, or even better the name of the nerve in full ie the mandibular division of the trigeminal nerve. This would help to prevent any future confusion.

It is also interesting to note that there is in fact an endothelial lining of the cavernous sinus ${ }^{1}$ meaning VI (abducent) and the internal carotid artery do not 'run free within the venous blood of the cavernous sinus', nor 'course through the venous blood' as was suggested in Professor Dean's original letter.

S. Lumley

By email

1. Standring S (Ed). Gray's anatomy. 39th edn. p 280. Fig. 15.6. Oxford: Elsevier Churchill Livingstone, 2005.

doi: 10.1038/bdj.2007.330

\section{Key to prevention}

Sir, I am writing in regard to the Opinion paper, Written off, by David Croser (BDJ 2006; 201: 497-499). I have followed with great interest the ensuing letters on this issue, all in agreement with Dr Croser, except perhaps the UKAP response. Drs Marianos and Reznik presented a well-reasoned and evidence-based argument against the current UKAP policy. In particular that the risk of transmission of HIV from an infected provider to a patient is extremely low as evidenced by only one case report of transmission to six patients in the Florida event associated with Dr Acer. Lookback studies by the Centers for Disease Control and Prevention (CDC) on 22,032 patients of $63 \mathrm{HIV}$-infected providers (including 33 dentists/dental students) found no other evidence of HIV transmission to a patient. Many rushed to judgement in the United States relative to 
the Acer case, including members of our Congress. Policy was put forward that was based in emotion, not in science. Wisdom having prevailed, the CDC recommendations for preventing transmission of human immunodeficiency virus and hepatitis B virus to patients during exposure-prone invasive procedures, which were issued prior to the Acer case, were deemed sufficient. Our legislators mandated that states, within one year, certify to the CDC their compliance with these recommendations or submit their own plan for approval. All states and territories complied with this. No other transmission has been reported. Policy went from emotional response to reliance on the sound medical judgement of experts.

What remains disturbing however is the UKAP acknowledgement of poor compliance with infection control in dental settings. Should the UKAP desire to make efforts to enhance the safe delivery of oral health services and to minimise the risk of transmission of bloodborne pathogens by provider to patient, patient to provider, or between patients, then regulating or mandating universal compliance with standard precautions would be indicated. CDC has emphasised that compliance with recommended infection control practices is the key to prevention of disease transmission. Standard precautions, including the use of safe devices combined with safe work practice, are the most protective mechanism offered. All dental practices must adhere to standard precautions, perform self-assessments to identify the practices and behaviours that put providers and patients at risk, modify these procedures, train staff on a regular basis, report and manage injuries. I would strongly suggest that this be the direction of the policy in the UK and the restrictive nature of removing HIV-infected dental healthcare workers be reconsidered.

H. Bednarsh

Director HIV Dental -

Boston Public Health Commission

Vice President HIVDENT

doi: 10.1038/bdj.2007.331

\section{A worldwide issue}

Sir, I have read with interest the recent views expressed in your journal about the infected dental health care worker (DHCW).

Appropriate management of the infected dental health care worker is, as has been stated, a worldwide issue. Our Dental Board in Victoria currently oversees some practitioners with blood-borne viruses and, in my view, this issue is one in which a pragmatic evidence-based approach is of benefit to both the public and to the infected DHCW.

A lot of the controversy over the issue of infected DHCWs continuing to practice seems to centre on what an exposure prone procedure is. Some 15 or more years ago, Australian dentistry adopted a procedure-based list of exposure prone procedures. The unjustness and impracticality of such an approach soon became apparent, and this highly restrictive list was abandoned in favour of assessing the real risk to the patient.

So, an individual assessment of each practitioner was conducted by an expert panel. Based on careful assessment of the practitioner's skills and expertise, the use of standard precautions and risk reduction procedures, the actual infectivity of the DHCW based on viral DNA/RNA levels, it has been possible to allow these infected DHCWs to continue practising, sometimes with procedure restrictions, and sometimes under some form of ongoing monitoring.

This approach allows practitioners to remain productive to the community in a time of severe shortage of dentists and other dental health care workers; we are not aware that any look-backs in recent times have linked transmission of HIV, Hepatitis B or C to an infected dental health care worker.

I wish my dental colleagues in the UK well as they continue this debate.

\section{G. Condon}

President, Dental Practice Board of Victoria, Australia doi: 10.1038/bdj.2007.332

\section{Pity the GDP}

Sir, Poorly managed dental pain (BDJ 2007; 202: 203-206) highlights problems within the current imposed NHS contract. Messrs Porter and Poyser are unlikely to have had experience working with the new contract and its associated treadmill, and so are perhaps a bit high-handed with their liberal criticism of the lady's dental experiences (who had no regular dentist and yet sought an immediate appointment from a random GDP).

To see this lady, take medical and dental histories, carry out a clinical examination, take appropriate radiographs, develop review and report on these, diagnose, anaesthetise and carry out treatment having obtained informed consent, is rewarded with 1.2 UDAs. Our Chief Dental Officer feels that 6 UDAs per hour should be achieved, so this 'emergency treatment' has a time-slot of 12 minutes attached to it, including swabbing down the surgery for the next patient.
Pity then, the poor random GDP - criticise the system he has to work under, if you like - but answer, honestly, could you, safely and reliably, diagnose and treat this lady's pain in 12 minutes? Would you also be comfortable completing this RCT, possibly carrying out an RCT in the LL6 and providing permanent restorations in those teeth and the UR6 in the 30 minutes you have for your 3 UDAs?

P. N. Burnell

By email

doi: 10.1038/bdj.2007.333

\section{Divided and ruled}

Sir, I would like to congratulate you on your editorial A profession no longer (BDJ 2007; 202: 235). Thanks to our colleague Dr Shipman, the medical and dental professions will never be the same again. Our reputations are in tatters and slavery to future governments is now written on our foreheads in bold letters. You are right: it is, as in dictatorial states, that without any consultation not only will the control of the GDC pass into the Government's hands, but we will pay for it. We are going to be divided and ruled. The fear of losing your livelihood is perhaps more important than any remaining self respect. We must have been blind or we should have seen it coming. Every time the Government wants to beat us with a stick all they have to do is to wave a banner with Shipman written on it. The Government probably had enough of us after the contract fiasco and also thinks that we will accept any other imposition just as well. I. A. S. Syed

By email

doi: 10.1038/bdj.2007.334

\section{A vain hope}

Sir, I read with interest the case report from Porter et al. (A life threatening event from poorly managed dental pain BDJ 2007; 202: 203-206) but feel the conclusions that "the recently introduced new contract for NHS general dental practitioners may help improve the problem of access to emergency dental care' is unfortunately a vain hope. The opposite is more likely to be the case for two important reasons. One is to do with the regulatory framework upon which the new contract is built and the other is the profession's behavioural response to the new contract.

The definition of a patient in the regulations is 'a person to whom the contractor is proving services under the contract' ${ }^{1}$ [my italics]. The key aspect of this is the fact that a patient is only a patient for the duration of a course of 
treatment, be that single or multiplevisits and that upon the termination of that course of treatment the patient is no longer entitled to care from that practitioner even if the last visit was the previous day. For all intents and purposes they become a member of the public again. The stark reality is that patients have fewer rights under the present contract than under the old one. There is therefore, perversely, no contractual obligation on an NHS practitioner to see a person in pain since neither registration exists, nor is there any form of continuing care relationship. Unless a practitioner has a specific service level agreement to provide access slots to see patients in pain, no obligation exists to see the types of patient this case report features. When a practice runs out of UDAs, as many have done in the weeks and months running up to the end of the first financial year of the contract, this effect is compounded.

The second reason that sadly makes the likelihood of other cases such as this possible is the behavioural response of the profession to the contract. There is no doubt the operation of the contract is fundamentally affected by the activity driven measure of UDAs but it is also affected by the practitioner's response to it. NHS dentists are trying to make the contract work but in doing so may inadvertently compromise the care of patients.

Irrespective of the vagaries of the NHS system in which we work, we owe it to people in pain to try and see them, do our best to diagnose their problems and even if we are unable to provide the definitive treatment, at least relieve them of pain no matter how the new NHS contract appears to relieve us of this obligation. We at least owe that to the public we serve from an ethical, professional and moral perspective.

L. D'Cruz

Woodford Green

1. The National Health Service (General Dental Services Contracts) Regulations 2005.

doi: 10.1038/bdj.2007.335

\section{A backward step}

Sir, is it not a little odd that as the House of Lords seems set to move to an all elected body, the GDC will become all appointed? This hardly seems progress and one could speculate that the next step in the evolution of the GDC will be the creation of hereditary members.

\section{J. R. Drummond}

Dundee

doi: 10.1038/bdj.2007.336
A multi-professional team

Sir, following my successful experiences working with a dental therapist ( $B D J$ 2006; 200: 193-197), I wonder whether more could be done to enable therapists to sit more comfortably in general practice in the new NHS, where UDAs are the only currency and where in most cases there is no way of measuring the therapist's contribution to the practice workload.

The delivery of practice UDAs is, in the most part, seen as an individual rather than a group effort. Employing a therapist who undertakes most of the routine dentistry means that the dentist is constantly undertaking more complex procedures which, for the dentist, is not a good way of achieving a high UDA target. In addition, there are no financial incentives in referring to a therapist as most performers will incur a financial penalty if they do not achieve their UDA target and within most practices where they are not the provider, money will be docked from their income to pay for the therapist. One solution to this would be for the PCTs to commission directly from the therapist.

In my pilot site the therapist managed very successfully an undifferentiated case load within the list of permitted duties laid down by the GDC. This has now been repealed and a therapist can now undertake practice procedures for which they have been trained and have experience. According to the BDA recent therapy courses vary on how well they have prepared the therapists for their duties and the GDC has not yet approved training programmes for any additional duties.

Ross et al. in their recent article ( $B D J$ 2007; 202: E8) report that dentists have only limited knowledge of a therapist's clinical remit and not surprisingly, this leads to unease, when the dentists have vicarious responsibility for the employed therapist's acts and omissions.

In order to provide solutions to these situations, funding streams need to be identified so that successful multi-disciplinary courses that emphasise the benefits of working as a practice team can be set up. Here, focusing on the dental team as a multi-professional unit, the professional, managerial and health and safety issues involved in working together in practice can be addressed. In addition, Personal Development Plans for therapists should be put in place and postqualifying training needs to be quality assured and performance managed.

P. Ward

Nottingham

doi: $10.1038 /$ bdj.2007.337 